\title{
The effect of mindfulness meditation on time perception
}

\section{Robin S. S. Kramer ${ }^{\mathrm{a}, *}$, Ulrich W. Weger ${ }^{\mathrm{b}}$, Dinkar Sharma ${ }^{\mathrm{a}}$}

${ }^{a}$ School of Psychology, Keynes College, University of Kent, Canterbury CT2 7NP, Kent, UK ${ }^{\mathrm{b}}$ Department für Psychologie und Psychotherapie, Universität Witten/Herdecke, AlfredHerrhausen-Straße 50, 58448 Witten, Germany

* Corresponding author. Address: School of Psychology, Keynes College, University of Kent, Canterbury CT2 7NP, Kent, UK

E-mail address: R.S.S.Kramer@kent.ac.uk (R. S. S. Kramer). 


\begin{abstract}
Research has increasingly focussed on the benefits of meditation in everyday life and performance. Mindfulness in particular improves attention, working memory capacity, and reading comprehension. Given its emphasis on moment-to-moment awareness, we hypothesised that mindfulness meditation would alter time perception. Using a within-subjects design, participants carried out a temporal bisection task, where several probe durations are compared to "short" and "long" standards. Following this, participants either listened to an audiobook or a meditation that focussed on the movement of breath in the body. Finally, participants completed the temporal bisection task for a second time. The control group showed no change after the listening task. However, meditation led to a relative overestimation of durations. Within an internal clock framework, a change in attentional resources can produce longer perceived durations. This meditative effect has wider implications for the use of mindfulness as an everyday practice and a basis for clinical treatment.
\end{abstract}

Keywords: Mindfulness, Meditation, Time perception, Temporal bisection, Attention, Arousal

\title{
1. Introduction
}

Research over the last few decades has begun to explore the effects of traditional Buddhist practices that have been around for millennia. Mindfulness, originally defined as having awareness, attention, and remembering (Bodhi, 2000), is one such example. Practitioners learn to focus their attention on both external and internal sensory stimuli with a non-judgmental awareness of the present moment (Kabat-Zinn, 2003). The goals of such a practice include improved metacognitive awareness, decreased rumination through a reduction in perseveration, and enhanced attention through gains in working memory (Davis \& Hayes, 2011). Important for the current research is the idea that mindfulness meditation can help practitioners to focus their attention on moment-tomoment awareness, and that this attention can be directed internally (such as on one's breathing) as well as externally. An enhanced moment-to-moment awareness might be expected to alter our sense of time, given the increased focus on the 'here and now'. However, few studies to date have considered the effects of such practices on time perception, i.e., how mindfulness meditation might affect the subjective passage of time.

Investigators have started to identify the benefits of mindfulness and mindfulness-based therapies in a variety of domains. These include decreases in rumination (Chambers, Lo, \& Allen, 2008), improvements in cognitive flexibility (Moore \& Malinowski, 2009), working memory capacity and sustained attention (Chamber et al., 2008; Jha, Stanley, Kiyonaga, Wong, \& Gelfand, 2010; MacLean et al., 2010; Mrazek, Franklin, Phillips, Baird, \& Schooler, 2013), and reductions in reactivity (Cahn \& Polich, 2009), anxiety and depressive symptoms (Hoffman, Sawyer, Witt, \& Oh, 2010). Indeed, mindfulness-based treatments appear to provide broad antidepressant and antianxiety effects, as well as decreases in general psychological distress (Marchand, 2012). As such, these interventions have been applied with a variety of patients, including those suffering from fibromyalgia, psoriasis, cancer, binge eating, and chronic pain, although the efficacy of specific methods requires further investigation (see meta-analysis by Baer, 2003).

While researchers have begun to uncover the effects of mindfulness on cognitive processes like working memory or attention, there has been little experimental consideration with regard to how mindfulness practice may affect the perception of time. The most common models used within the field of time perception posit a single internal clock, which includes a pacemaker (Church, 1984; Treisman, Faulkner, Naish, \& Brogan, 1990) and an attentional gate (Zakay \& Block, 1997). In these models, the pacemaker is responsible for emitting pulses, while the attention-controlled switch closes at the onset, and opens at the offset, of a stimulus, allowing pulses to enter an 
accumulator. Time estimation is based on the number of pulses accumulated, with more pulses leading to an increase in perceived duration. Arousal produces an overestimation of time due to an increase in the pacemaker's speed (Maricq, Roberts, \& Church, 1981; Meck, 1983; Wearden \& Penton-Voak, 1995; although see Lui, Penney, \& Schirmer, 2011). In contrast, if attention to the task distracts from the processing of temporal information, this opens the switch and some pulses are lost. The result is an underestimation of time (e.g., Tipples, 2010; for a review, see Lejeune, 1998). Importantly, meditation appears to manipulate both attention and arousal level (e.g. West, 1987). Glicksohn's (2001) modification of the above model aimed to incorporate the potential effects of meditation. Here, perceived duration is defined as the product of the number of subjective time units (synonymous with pulses in the internal clock model) and the size of these units. During focussed meditation, there is an increase in internally oriented attention and a reduction in arousal (Schuman, 1980). A more efficient allocation of attentional resources to internal stimulation is thought to decrease the number of subjective time units (due to a decrease in arousal, much like the original model) and increase the size of the subjective time units (Zakay, 1989). Consequently, there is an increase in perceived duration and the flow of time becomes slower.

Mindfulness training involves deliberately staying in the present moment for as long and as continuously as possible (Hollis-Walker \& Colosimo, 2011). Indeed, researchers found that mindfulness meditation experts were able to stabilise a bistable image (the Necker Cube) for longer in comparison with non-meditators, suggesting a longer duration of subjective nowness (Sauer et al., 2012). This result can be explained by evidence suggesting that mindfulness meditation trains attentional skills and produces increased attentional resources (Lutz, Slagter, Dunne, \& Davidson, 2008). If mindfulness meditators are able to attend to a given task while still being able to apply these increased resources to the processing of temporal information, this would close the internal clock's switch and accumulate additional pulses (in terms of the conventional model above). Alternatively, the ability to increase the amount of internally oriented attention would increase the size of subjective time units (modified model). In both cases, we might therefore hypothesise a resulting overestimation of time. In the only previous study on this topic, participants were asked to produce specified target durations by pressing a finger button, and mindfulness meditation practitioners produced longer durations than controls (Berkovich-Ohana, Glicksohn, \& Goldstein, 2012). This is equivalent to participants underestimating durations since the slower accumulation of pulses (or time units) would cause participants to wait longer before responding that a target duration had passed, but would feel like an experienced duration had lasted for a shorter amount of time. Longer produced durations may be explained by a decrease in arousal (due to a decrease in pacemaker speed), potentially combined with the hypothesised increase in size of the subjective time units.

In the current experiment, we employed the temporal bisection task, which was developed to test the predictions of clock-based models (e.g., Chambon, Droit-Volet, \& Niedenthal, 2008). In a training phase, participants learn two standard durations, one short and one long. In the testing phase, they are presented with comparison stimulus durations (the standards and also intermediate durations). The task is to classify each comparison duration as more similar to either the short or the long standard duration. If mindfulness meditation produces an overestimation of time, this should lead participants who carry out a meditation task to more often classify the comparison durations as similar to the long standard (compared with their performance prior to meditation). In contrast, performance for participants in a control group should remain unchanged.

\section{Materials and methods}

\subsection{Participants}


Forty undergraduate students from the University of Kent (age range, 18-24 years; 35 females) participated in exchange for course credits.

\subsection{Design}

The experiment was defined by five factors that described the testing and stimuli: Group (control or meditation) x Session (first or second) x Shape (circle or square) x Colour (red, green, blue) x Duration (400, 600, 800, 1000, 1200, 1400, 1600ms). Group varied between participants, while all other factors varied within participants. The dependent measure was the proportion of "long" responses (see Section 2.4).

\subsection{Materials}

Participants were tested individually in a quiet laboratory room, with an electric fan providing background 'white noise'. The experiment was presented on a Dell laptop computer using custom MATLAB software to control the presentation of the experimental stimuli and to record the participants' responses. The stimulus used for the representation of duration during training blocks was a grey oval $(3.5 \times 7 \mathrm{~cm})$. During the testing block, squares $(5 \times 5 \mathrm{~cm})$ and circles $(5.5 \mathrm{~cm}$ diameter) with identical surface areas, saturation, and brightness were presented in three different colours (RGB values in square brackets): red [ [230 0 0], green [ [ 230 0], and blue [ $\left[\begin{array}{lll}0 & 0 & 230\end{array}\right]$. Each shape was presented in the centre of the screen on a black background.

Participants completed basic demographic information along with two measures of mindfulness during everyday life. The Mindfulness Attention Awareness Scale (MAAS; Brown \& Ryan, 2003) consists of 15 items that are rated on a 6-point Likert scale from 1 (almost always) to 6 (almost never), where the mean rating across all items represents the final score, with higher scores reflecting greater mindfulness. The Five-Factor Mindfulness Questionnaire (FFMQ; Baer, Smith, Hopkins, Krietemeyer, \& Toney, 2006) consists of 39 items that are rated on a 5-point Likert scale from 1 (never or very rarely true) to 5 (very often or always true). Mean ratings (after reverse scoring specific items) are calculated for each of five facets: observing, describing, acting with awareness, non-judging of inner experience, and non-reactivity to inner experience. Again, higher scores reflect greater mindfulness.

Given that the FFMQ is derived from a factor analysis of several questionnaires including the MAAS, we would predict at least some correlation between participants' scores on these instruments. However, since such relationships are far from perfect (Baer et al., 2006) and both measures remain popular, we decided to include both questionnaires in the current study.

In the listening task, participants in the control group listened to the beginning of the audiobook version of 'The Hobbit' (Shaw, 2005). Those in the meditation group listened to a 'mindfulness of body and breath' exercise (Williams \& Penman, 2011) designed to focus their attention on the movement of the breath in the body. We selected an audiobook as a control since it requires a comparable amount of attention and concentration to the meditation task, although the focus of attention in the two tasks was necessarily, and importantly, different. We decided that this better matched the meditation task in comparison with a mental arithmetic task (Hölzel et al., 2007) or no task at all. The audio recordings were presented using Beyerdynamic DT770 closed-back headphones.

\subsection{Procedure}

Participants were informed through a printed information sheet that the experiment investigated "individual differences in how we judge the passage of time". They first completed both questionnaires along with demographic information. Following this, participants performed a 
temporal bisection task (first session) composed of two successive phases: training and testing. In the training phase, participants were shown the short and long stimulus durations, represented by the grey oval stimulus. The short standard stimulus duration (S) was $400 \mathrm{~ms}$ and the long one (L) $1600 \mathrm{~ms}$. Each standard was initially presented five times in alternation. Participants responded by pressing the " $d$ " and " $k$ " keys on the computer keyboard after the short and the long standard durations. The key-response match was counterbalanced across participants. Next, each participant performed a block of trials in which $\mathrm{S}$ and $\mathrm{L}$ had $50 \%$ probability of appearing on each trial. This block terminated only after participants responded correctly on eight consecutive trials. Throughout training, the inter-trial interval was randomly chosen between $1 \mathrm{~s}$ and $3 \mathrm{~s}$. Accuracy feedback, positive ("correct") or negative ("incorrect"), was presented for $2 \mathrm{~s}$ in the centre of the computer screen. The experimenter remained with the participant to make sure there were no difficulties during training, but the participant was alone during the testing phase.

In the testing phase, the participants were presented with coloured squares and circles that represented the seven comparison durations $(400,600,800,1000,1200,1400$, and 1600ms). Their task was to judge whether the duration of each presented shape was closer to S or L. Each combination of shape (circle, square) and colour (red, green, blue) was presented three times for each comparison duration, producing a total of 126 trials. The trials were presented in a random order with no accuracy feedback, and the inter-trial interval was randomly chosen between $1 \mathrm{~s}$ and $3 \mathrm{~s}$. Viewing distance was approximately $60 \mathrm{~cm}$ but was not fixed.

On completion, participants then listened to a $10 \mathrm{~min}$ audio recording with instructions to follow along as best they could, and to inform the experimenter when it finished. Those in the meditation group were presented with a breathing exercise while those in the control group listened to a neutral recording (see Section 2.3). The assignment of participants to groups alternated, based upon when they took part.

After the listening task, participants completed the temporal bisection task again (second session). The procedure was identical to earlier, including the use of the training phase. As before, the experimenter remained with the participant during training, but the participant was alone during the testing phase. No breaks were provided between tasks.

\section{Results}

\subsection{Preliminary analyses}

Participants' scores on the MAAS and FFMQ were calculated, and the two groups (control vs. meditation) were subsequently compared. Participants in the two groups did not differ in age, MAAS scores, or on any of the five factor scores (all $t \mathrm{~s}<1.26$, all $p \mathrm{~s}>.22$ ).

Prior to analysis of the temporal bisection data, the mean proportion of "long" responses was calculated for each of the six types of stimuli (two shapes and three colours) for each participant, separately for each of the two sessions ${ }^{1}$. In addition, to allow exploration of the magnitude of the differences in temporal perception between the first and second sessions, the proportions of "long" responses for each participant for each stimulus duration and session (across all stimuli) were

\footnotetext{
${ }^{1}$ These proportions for both groups were entered into a five-way mixed analysis of variance including Group (2), Session (2), Shape (2), Colour (3), and Duration (7). The predicted Group x Session interaction approached significance, $F(1,38)=2.85, p=.100$. However, this interaction lacked statistical power (.39), and given that we hypothesised group differences regarding the influence of the listening task, we subsequently analysed the two groups separately.
} 
transformed with the probit function to $z$-scores ${ }^{2}$. Then, an index of difference $d^{\prime}$ was calculated by subtracting the $z$-score for the first session from the $z$-score for the second session (Macmillan \& Creelman, 1991), yielding $7 d^{\prime}$ scores for each participant. These $d^{\prime}$ values are positive if the temporal stimulus was judged to match the longer standard more often in the second compared to the first session. Positive $d^{\prime}$ values thus indicate that stimulus duration was perceived as longer in the second session. Conversely, $d^{\prime}$ values are negative if the temporal stimulus was judged to match the longer standard less often in the second compared to the first session. Negative $d^{\prime}$ values thus indicate that stimulus duration was perceived as shorter in the second session.

Finally, the bisection point (BP; the point of subjective equality, i.e., the duration at which the proportion of "long" responses equals 0.5 ) was calculated for each participant for each session (across all trials). Although several methods exist for determining the BP, they tend to produce similar values (Wearden \& Ferrara, 1995). Here, we used the most straightforward method of interpolating the duration from the straight line joining the two data points that straddle the region where the 50\% "long" responses occurred (Raslear, 1983). The advantage of this method is that it involves no assumptions regarding the shape of the psychometric function, in contrast with linear regression methods.

Although we might predict that participants' BPs would demonstrate a relationship with their trait mindfulness scores on the MAAS and the FFMQ, we found no significant correlations with their BPs during the first or second sessions (all $p \mathrm{~s}>.258$ ). Participants' BP differences (second session minus first) also showed no relationship with questionnaire scores (all $p s>$.149). Finally, these analyses were carried out for the two groups separately, again producing no significant correlations (all $p \mathrm{~s}>.083$ ).

\subsection{Control group}

Fig. 1 shows the mean proportion of "long" responses plotted against duration for participants in the control group. These proportions were entered into a four-way within-participant analysis of variance including Session (2), Shape (2), Colour (3), and Duration (7). As expected, there was a significant main effect of Duration, $F(6,114)=239.45, p<.0001$, indicating that mean proportions varied across stimulus durations. There was no main effect of Session, $F(1,19)=2.53, p=.129$, and no other main effects or interactions were significant (all $F \mathrm{~s}<2.73$, all $p \mathrm{~s}>.078$ ).

To further examine whether stimulus durations differed in the two sessions, we used a onesample $t$-test to determine if the average $d^{\prime}$ was significantly different from zero. As expected, there was no significant difference, $t(19)=1.72, p=.103$. Finally, we used a paired-sample $t$-test in order to compare the BPs in the two sessions, and again found no significant difference, $t(19)=1.91, p=$ .072 . Indeed, neither the BPs in the first $(M=1024)$ nor the second $(M=958)$ session differed from $1000 \mathrm{~ms}$ (both $t \mathrm{~s}<1.13, p \mathrm{~s}>.271$ ), which represents the halfway point between the short and long standard stimulus durations.

\subsection{Meditation group}

Fig. 2 shows the mean proportion of "long" responses plotted against duration for participants in the meditation group. These proportions were entered into a four-way within-participant analysis of variance as above. As expected, there was a significant main effect of Duration, $F(6,114)=$ $264.15, p<.0001$, indicating that mean proportions varied across stimulus durations. There was

\footnotetext{
${ }^{2}$ Although $z$-scores sometimes refer to standardized scores, signal detection theory defines $z$ as the inverse function of the normal distribution curve (Macmillan \& Creelman, 1991), which is how the term is used here. Also, in order for this transformation to be accomplished on proportions, values of 1 and 0 had to be replaced with a high and low decimal, respectively. We substituted .944 for 1, and .056 for 0 in line with previous research (e.g., Effron, Niedenthal, Gil, \& Droit-Volet, 2006).
} 
also a main effect of Session, $F(1,19)=27.40, p<.0001$, indicating that the proportion of "long" responses was greater in the second session $(M=0.564)$ compared with the first $(M=0.477)$. These results were qualified by a significant Session x Duration interaction, $F(6,114)=6.19, p<.0001$. Pairwise comparisons revealed that the proportion of "long" responses was significantly greater in the second session for all stimulus durations $(600,800,1000,1200,1400 \mathrm{~ms})$ other than the two standards (400, 1600ms) (all $p \mathrm{~s}<.021$ after Bonferroni correction). Finally, the main effect of Shape was close to significant, $F(1,19)=3.82, p=.065$, although we have no sensible explanation for this and will not pursue it further here. No other main effects or interactions were significant (all $F_{\mathrm{s}}<1.56$, all $\left.p \mathrm{~s}>.105\right)$.

To further examine whether stimulus durations differed in the two sessions, we used a onesample $t$-test to determine if the average $d^{\prime}$ was significantly different from zero. As expected from the above results, there was a significant difference, $M=0.28 ; t(19)=5.40, p<.0001$. This reinforced the idea that participants overestimated the stimulus durations in the second session compared with the first.

Finally, we used a paired-sample $t$-test in order to compare the BPs in the two sessions, and found a significant difference, $t(19)=5.05, p<.0001$. The BPs in the first session $(M=998)$ did not differ from $1000 \mathrm{~ms}, t(19)=0.08, p=.936$. In contrast, the BPs in the second session $(M=876)$ were significantly lower than $1000 \mathrm{~ms}, t(19)=4.13, p=.001$.

An overestimation of stimulus durations in the second session compared with the first, combined with a decrease in BPs, might be interpreted within an arousal framework. An increase in arousal is most often associated with an increase in pacemaker speed, and this, in turn, increases the number of pulses accumulated within a given time period and produces an overestimation. However, this increase in pacemaker speed would produce a multiplicative effect, i.e., an increase in the magnitude of the time distortion as the length of stimulus durations increased (Maricq et al., 1981; Meck, 1983). In order to test this hypothesis, we compared the average $d$ ' of the shortest comparison durations $(400,600$, and $800 \mathrm{~ms})$ with the average $d^{\prime}$ of the longest comparison durations (1200, 1400, and $1600 \mathrm{~ms})$, with an arousal account predicting an increase in $d^{\prime}$ for longer durations (Gil, Niedenthal, \& Droit-Volet, 2007). However, we found no significant difference, $t(19)=1.12, p=.275$. As such, an account based upon increased pacemaker speed was not supported.

\section{Discussion}

The present experiment showed that listening to a mindfulness meditation exercise, which focussed one's attention on the movement of the breath in the body, led participants to classify stimulus durations as "long" more often in comparison with prior to this exercise. In contrast, participants who listened to a neutral recording (an audiobook) showed no change in their responses in comparison with before the task.

With regard to internal-clock models, an overestimation of duration is often explained through an increase in arousal (an increase in pacemaker speed increases the number of pulses accumulated), while an underestimation is usually associated with attentional mechanisms (the task's attentional demands cause the switch to open and pulses are lost). Although meditation in this experiment produced an overestimation, we interpret the current results within an attentional framework for several reasons. First, we found no evidence of a multiplicative effect due to arousal, whereby increased pacemaker speed produces larger overestimates of duration as the length of the stimulus duration increases. Second, mindfulness meditation is widely accepted as producing a reduction in stress and arousal (e.g., Chang et al., 2004; Chu, 2010; Cincotta, Gehrman, Gooneratne, \& Baime, 2011) rather than an increase. Third, previous research has already established a link between focussed attention during meditation and activation in multiple brain regions associated with attention/monitoring (e.g. Brefczynski-Lewis, Lutz, Schaefer, Levinson, \& 
Davidson, 2007; Dickenson, Berkman, Arch, \& Lieberman, 2013). Therefore, participants' overestimations are more likely the result of attentional changes, producing either improved attentional resources that allow increased attention to temporal processing, or a shift to internally oriented attention that may increase the size of the subjective time units (Glicksohn, 2001). Teasing these two accounts apart is beyond the scope of the current research but provides an interesting path for future studies.

By asking participants to follow a 10min mindfulness meditation, our manipulation focussed on what is likely a temporary change in perception. While mindfulness practice (which can be considered a state; Davis \& Hayes, 2011) aims to increase one's ability to remain mindful during everyday life (trait mindfulness), the relationship between these two constructs is far from clear (Thompson \& Waltz, 2007). Indeed, our results found no relationship between trait mindfulness and individual performance on the temporal bisection task, although a larger sample size may provide the statistical power needed to detect what are likely to be relatively small effects. Certainly, an interesting consideration for future research would be to investigate whether individuals who score higher on trait-based mindfulness measures show a difference in their perceptions of time (and hence their response bias) in comparison with low scorers. Related, we might also consider how long our demonstrated change in time perception lasts, and so begin to explore the development from state to trait mindfulness.

In the present experiment, an unselected sample of undergraduate students demonstrated a significant meditation effect despite a lack of prior training. While typical participants usually receive professional training through the attendance of meditation classes or retreats (e.g., Mrazek et al., 2013), only a recorded exercise was provided here. As a result, our participants also received a comparatively short meditation experience (ten minutes, rather than many hours or days). Taken together, these aspects demonstrate that subjective time is very sensitive to even short meditative states and the changes these induce, even for those who are not meditation experts. This result has useful implications for future research and also treatment protocols.

We predict that the type of mindfulness meditation used is an important factor in the resulting change to the internal clock. Here, we featured a breathing exercise that aimed to focus participants' attention on the movement of the breath in the body. Such an internally oriented task, involving focussed attention, may produce different results compared with an open monitoring meditation, for example, where moment-to-moment attention is not focussed on any explicit object. Indeed, researchers showed that activation linked with compassion meditation (which encourages a state of loving kindness and compassion towards all beings) related to brain circuitry involved with emotion, empathy, and theory of mind (Lutz, Brefczynski-Lewis, Johnstone, \& Davidson, 2008). As such, it may be that other types of meditation can lead to increased arousal or an increase in attention to external events.

In conclusion, the present findings represent some of the first to demonstrate how mindfulness meditation can alter the perception of time, and the first regarding time estimation. We also show that the temporal bisection task is ideally suited to an in-depth investigation of the mechanisms underlying this effect. Given the increasing popularity of mindfulness both in everyday practice and as a recognised basis for clinical treatment, its relationship with time perception may provide an important step in our understanding of this pervasive, ancient practice in our modern world.

\section{Acknowledgments}

This work was supported by grant F/00 236/AB from the Leverhulme Trust to Ulrich Weger and Dinkar Sharma. The authors would like to thank Alex Jones for creation of the figures, along with Joseph Glicksohn and an anonymous reviewer for their helpful comments.

\section{References}


Baer, R. A. (2003). Mindfulness training as a clinical intervention: A conceptual and empirical review. Clinical Psychology: Science and Practice, 10, 125-143.

Baer, R. A., Smith, G. T., Hopkins, J., Krietemeyer, J., \& Toney, L. (2006). Using self-report assessment methods to explore facets of mindfulness. Assessment, 13, 27-45.

Berkovich-Ohana, A., Glicksohn, J., \& Goldstein, A. (2012). Mindfulness-induced changes in gamma band activity - Implications for the default mode network, self-reference and attention. Clinical Neurophysiology, 123, 700-710.

Bodhi, B. (2000). A comprehensive manual of Adhidhamma. Seattle: BPS Pariyatti.

Brefczynski-Lewis, J. A., Lutz, A., Schaefer, H. S., Levinson, D. B., \& Davidson, R. J. (2007). Neural correlates of attentional expertise in long-term meditation practitioners. Proceedings of the National Academy of Sciences USA, 104, 11483-11488.

Brown, K. W., \& Ryan, R. M. (2003). The benefits of being present: Mindfulness and its role in psychological well-being. Journal of Personality and Social Psychology, 84, 822-848.

Cahn, B. R., \& Polich, J. (2009). Meditation states and traits: EEG, ERP, and neuroimaging studies. Psychological Bulletin, 132, 180-211.

Chambers, R., Lo, B. C. Y., \& Allen, N. B. (2008). The impact of intensive mindfulness training on attentional control, cognitive style, and affect. Cognitive Therapy and Research, 32, 303-322.

Chambon, M., Droit-Volet, S., \& Niedenthal, P. M. (2008). The effect of embodying the elderly on time perception. Journal of Experimental Social Psychology, 44, 672-678.

Chang, V. Y., Palesh, O., Caldwell, R., Glasgow, N., Abramson, M., Luskin, F., \& Koopman, C. (2004). The effects of a mindfulness-based stress reduction program on stress, mindfulness self-efficacy, and positive states of mind. Stress and Health, 20, 141-147.

Chu, L. (2010). The benefits of meditation vis-à-vis emotional intelligence, perceived stress and negative mental health. Stress and Health, 26, 169-180.

Church, R. M. (1984). Properties of the internal clock. In J. Gibbon \& L. Allan (Eds.), Timing and time perception (pp. 566-582). New York, NY: New York Academy of Sciences.

Cincotta, A. L., Gehrman, P., Gooneratne, N. S., \& Baime, M. J. (2011). The effects of a mindfulness-based stress reduction programme on pre-sleep cognitive arousal and insomnia symptoms: A pilot study. Stress and Health, 27, 299-305.

Davis, D. M., \& Hayes, J. A. (2011). What are the benefits of mindfulness? A practice review of psychotherapy-related research. Psychotherapy, 48, 198-208.

Dickenson, J., Berkman, E. T., Arch, J., \& Lieberman, M. D. (2013). Neural correlates of focused attention during a brief mindfulness induction. Social Cognitive and Affective Neuroscience, $8,40-47$.

Effron, D. A., Niedenthal, P. M., Gil, S., \& Droit-Volet, S. (2006). Embodied temporal perception of emotion. Emotion, 6, 1-9.

Gil, S., Niedenthal, P. M., \& Droit-Volet, S. (2007). Anger and time perception in children. Emotion, 7, 219-225.

Glicksohn, J. (2001). Temporal cognition and the phenomenology of time: A multiplicative function for apparent duration. Consciousness and Cognition, 10, 1-25.

Hoffman, S. G., Sawyer, A. T., Witt, A. A., \& Oh, D. (2010). The effect of mindfulness-based therapy on anxiety and depression: A meta-analytic review. Journal of Consulting and Clinical Psychology, 78, 169-183.

Hollis-Walker, L., \& Colosimo, K. (2011). Mindfulness, self-compassion, and happiness in nonmeditators: A theoretical and empirical examination. Personality and Individual Differences, $50,222-227$.

Hölzel, B.K., Ott, U., Hempel, H., Hackl, A., Wolf, K., Stark, R., \& Vaitl, D. (2007). Differential engagement of anterior cingulate and adjacent medial frontal cortex in adept meditators and non-meditators. Neuroscience Letters, 421, 16-21. 
Jha, A. P., Stanley, E. A., Kiyonaga, A., Wong, L., \& Gelfand, L. (2010). Examining the protective effects of mindfulness training on working memory capacity and affective experience. Emotion, 10, 54-64.

Kabat-Zinn, J. (2003). Mindfulness-based interventions in context: Past, present, and future. Clinical Psychology: Science and Practice, 10, 144-156.

Lejeune, H. (1998). Switching or gating? The attentional challenge in cognitive models of psychological time. Behavioral Processes, 44, 127-145.

Lui, M. A., Penney, T. B., \& Schirmer, A. (2011). Emotion effects on timing: Attention versus pacemaker accounts. PLoS ONE, 6(7), e21829.

Lutz, A., Brefczynski-Lewis, J., Johnstone, T., \& Davidson, R. J. (2008). Regulation of the neural circuitry of emotion by compassion meditation: Effects of meditative expertise. PLoS ONE, 3, e1897.

Lutz, A., Slagter, H., Dunne, J. D., \& Davidson, R. J. (2008). Attention regulation and monitoring in meditation. Trends in Cognitive Sciences, 12, 163-169.

MacLean, K. A., Ferrer, E., Aichele, S. R., Bridwell, D. A., Zanesco, A. P., Jacobs, T. L., ... Saron, C. D. (2010). Intensive meditation training improves perceptual discrimination and sustained attention. Psychological Science, 21, 829-839.

Macmillan, N. A., \& Creelman, C. D. (1991). Detection Theory: A user's guide. New York: Cambridge University Press.

Marchand, W. R. (2012). Mindfulness-based stress reduction, mindfulness-based cognitive therapy, and Zen meditation for depression, anxiety, pain, and psychological stress. Journal of Psychiatric Practice, 18, 233-252.

Maricq, A. V., Roberts, S., \& Church, R. M. (1981). Methamphetamine and time estimation. Journal of Experimental Psychology: Animal Behavior Processes, 7, 18-30.

Meck, W. H. (1983). Selective adjustment of speed of internal clock and memory processes. Journal of Experimental Psychology: Animal Behavior Processes, 9, 171-201.

Moore, A., \& Malinowski, P. (2009). Meditation, mindfulness and cognitive flexibility. Consciousness and Cognition, 18, 176-186.

Mrazek, M. D., Franklin, M. S., Phillips, D. T., Baird, B., \& Schooler, J. W. (2013). Mindfulness training improves working memory capacity and GRE performance while reducing mind wandering. Psychological Science. Advance online publication.

Raslear, T.G. (1983). A test of the Pfanzagl bisection model in rats. Journal of Experimental Psychology: Animal Behavior Processes, 9, 49-62.

Sauer, S., Lemke, J., Wittmann, M., Kohls, N., Mochty, U., \& Walach, H. (2012). How long is now for mindfulness meditators? Personality and Individual Differences, 52, 750-754.

Schuman, M. (1980). The psychophysiological model of meditation and altered states of consciousness: A critical review. In J. M. Davidson \& R. J. Davidson (Eds.), The psychobiology of consciousness (pp. 333-378). New York: Plenum.

Shaw, M. (Speaker). (2005). The Hobbit. [Digital recording]. London, UK: HarperCollins Audiobooks.

Thompson, B. L., \& Waltz, J. (2007). Everyday mindfulness and mindfulness meditation: Overlapping constructs or not? Personality and Individual Differences, 43, 1875-1885.

Tipples, J., (2010). Time flies when we read taboo words. Psychonomic Bulletin \& Review, 17, 563568.

Treisman, M., Faulkner, A., Naish, P. L. N., \& Brogan, D. (1990). The internal clock: Evidence for a temporal oscillator underlying time perception with some estimates of its characteristic frequency. Perception, 19, 705-743.

Wearden, J. H., \& Ferrara, A. (1995). Stimulus spacing effects in temporal bisection by humans. Quarterly Journal of Experimental Psychology B: Comparative and Physiological Psychology, 48, 289-310. 
Wearden, J. H., \& Penton-Voak, I. S. (1995). Feeling the heat: Body temperature and the rate of subjective time, revisited. Quarterly Journal of Experimental Psychology B: Comparative and Physiological Psychology, 48, 129-141.

West, M. A. (Ed.). (1987). The psychology of meditation. Oxford, UK: Oxford University Press.

Williams, M., \& Penman, D. (2011). Mindfulness: An eight-week plan for finding peace in a frantic world (Track 1). Oxford: Piatkus.

Zakay, D. (1989). Subjective time and attentional resource allocation: An integrated model of time estimation. In I. Levin \& D. Zakay (Eds.), Time and human cognition: A life-span perspective (pp. 365-397). Amsterdam: Elsevier.

Zakay, D., \& Block, R. A. (1997). Temporal cognition. Current Directions in Psychological Science, 6, 12-16. 